\title{
LAS INMISIONES DE OZONO EN LA CIUDAD DE ZARAGOZA, PERÍODO 2002-2008
}

\author{
Rosa Matute-Najarro' ${ }^{1}$ y José Carlos González-Hidalgo'-3 \\ 1 Dpto. de Ingeniería Química y TMA, Campus Actur, Zaragoza, España \\ rmature@unizar.es \\ 2 Dpto. de Geografía y OT, Universidad de Zaragoza \\ 3 Instituto Universitario de Ciencias Ambientales, Universidad de Zaragoza
}

\begin{abstract}
Resumen: Se han analizado las inmisiones de ozono de la ciudad de Zaragoza entre 2002-2008 a partir de los promedios diarios registrados en tres estaciones de seguimiento. La evolución interanual muestra el patrón de máximo de verano y mínimo de invierno, y en el periodo analizado muestra un aumento de las inmisiones. El calendario diario promedio permite observar las Diferencias entre el día y noche, que se acentúan en el verano. El estudio de los valores promedio diarios semanales refleja con claridad un aumento durante el fin de semana. En ningún momento los valores de inmisión han superado los umbrales legales establecidos, por lo que se puede afirmar que la ciudad no sufre problemas por este contaminante.
\end{abstract}

Palabras clave: Ozono troposférico, tendencias, calendario diario, calendario semanal, Zaragoza.

\begin{abstract}
Ozone concentration during 2002-2008 has been analyzed in Zaragoza. Interannual evolution shows the summer maximum-winter minimum pattern, and ozone inmission increased during the study period. Daily calendar shows daytime-nightime differences, accentuated in summer. Weekly daily values show the week-end effect. Ozone concentrations never reach the legal threshold. It can be said that ozone is not a pollutant dangerous in Zaragoza.
\end{abstract}

Keywords: Tropospheric ozone, trends, daily calendar, weekly calendar, Zaragoza.

Recibido: 2-11-10. Aceptado: 2-2-12. 


\section{Introducción}

Las ciudades son los espacios más humanizados del planeta, donde la contaminación del aire puede alcanzar su máxima intensidad y efectos. Por otro lado, el incremento de la población urbana durante todo el siglo XX ha originado un aumento del número de estudio de contaminación atmosféricas urbana por los efectos que pudiera tener sobre la población especialmente en áreas con grandes emisiones industriales, en zonas contaminadas, de climas cálidos y con valores elevados de radiación solar (Baldasano et al., 2003; Jiménez-Hornero et al., 2010). Dos de estas variables (temperatura y radiación) son características de las áreas de clima mediterráneo, donde localmente pueden además originarse precursores antropogénicos y biogénicos que favorezcan los procesos fotoquímicos que producen ozono (Gerasopoulos et al., 2006; Saínz-López et al., 2009).

Por la importancia que los precursores tienen sobre su formación, especialmente los derivados de las emisiones del tráfico, las máximas concentraciones cabría esperar que se concentrasen en zonas urbanas pero los movimientos atmosféricos pueden modificar esta situación (Jiménez-Hornero et al., 2010; Adame et al., 2010). Así, las concentraciones locales dependen, además, de las condiciones topográficas, el transporte atmosférico horizontal (con especial importancia en el régimen de brisas) y el transporte vertical y mezcla dentro de la troposfera y entre la troposfera y estratosfera (Adame et al., 2010).

Los efectos perniciosos del ozono se concentran en la salud humana (especialmente aparato respiratorio) y los ecosistemas. La exposición de corta duración a una concentración punta de ozono puede afectar de forma temporal a los pulmones, el tracto respiratorio y los ojos. También puede aumentar la susceptibilidad a los alérgenos respiratorios. Una exposición prolongada a concentraciones de ozono relativamente bajas también puede provocar una disminución de la función pulmonar. Por estas razones, desde hace años la Unión Europea dispone de una red de control de inmisiones que incluye numerosas ciudades, entre ellas Zaragoza, y entre cuyos contaminantes analizados se encuentra el ozono. En la legislación, vigente hasta el año 2003 y ya derogada (Directiva 92/72/CEE, transpuesta en el Real Decreto 1494/1995) se establecieron valores umbrales para la concentración en la atmósfera del ozono, siendo muy inferiores a los permitidos actualmente, tras la aprobación de la Directiva 2002/3/CE, de 12 de febrero, y a su correspondiente transposición al ordenamiento jurídico español en el Real Decreto 1796/2003, de 26 de diciembre, relativa al ozono en el aire ambiente. En esta nueva legislación se modificaron algunos umbrales de protección a la salud y se introdujeron nuevos estándares de calidad del aire en lo referente a concentración de ozono a medio y largo plazo. En la Tabla 1 se recogen los valores umbral actualmente en vigor. 
Tabla 1 Umbrales de Ozono según RD 1796/2003.

\begin{tabular}{|c|c|c|}
\hline Umbral & Valor & Período de referencia \\
\hline $\begin{array}{l}\text { Umbral de información } \\
\text { a la población }\end{array}$ & $180 \mu \mathrm{g} / \mathrm{m}^{3}$ & Promedio horario. \\
\hline $\begin{array}{l}\text { Umbral de alerta a la } \\
\text { población }\end{array}$ & $240 \mu \mathrm{g} / \mathrm{m}^{3}$ & $\begin{array}{l}\text { Promedio horario. Para los planes de } \\
\text { acción inmediata se evalúa durante } 3 \\
\text { horas consecutivas. }\end{array}$ \\
\hline Protección a la salud & $120 \mu \mathrm{g} / \mathrm{m}^{3}$ & $\begin{array}{l}\text { Media móvil octohoraria sin recuperación } \\
\text { máxima de cada día, no podrá superarse } \\
\text { más de } 25 \text { días por cada año civil de } \\
\text { promedio en un período de } 3 \text { años. }\end{array}$ \\
\hline $\begin{array}{l}\text { Protección a la } \\
\text { vegetación }\end{array}$ & $\begin{array}{l}\text { AOT } 40=6.000 \\
\mu \mathrm{g} / \mathrm{m}^{3} \mathrm{~h}\end{array}$ & Valores horarios de mayo a junio. \\
\hline Protección de los bosques & $\begin{array}{l}\text { AOT } 40=20.000 \\
\mu \mathrm{g} / \mathrm{m}^{3} \mathrm{~h}\end{array}$ & Valores horarios de abril a septiembre. \\
\hline Daños a los materiales & $40 \mu \mathrm{g} / \mathrm{m}^{3} \mathrm{~h}$ & Año civil. \\
\hline
\end{tabular}

Los estudios sobre ozono troposférico en España han abarcado diversos aspectos. Junto a los análisis de Millan et al., (2002) sobre sus precursores en la vertiente mediterránea, las condiciones atmosféricas y las concentraciones del contaminante se han estudiado en Madrid y su entorno (Fernández García, 2005; Palacios et al., 2002; Sánchez et al,, 2007), región norte (García et al., 2005), Bilbao (Ibarra y Madariaga, 2003) y en la Comunidad Valenciana (Castell et al., 2004a); en Barcelona SánchezLorenzo (2004) analizó su relación con otros contaminantes que pudieran ser sus precursores a lo largo de los días de la semana. Las concentraciones de primavera y verano y los ciclos diurnos y nocturnos fueron estudiados en Huelva por Adame et al., (2009), en Puertollano por Saiz-López et al., (2009) o en espacios subregionales como la Comunidad Valenciana por Castell et al., (2004b), mientras la estructura fractal de sus series temporales fue analizada por Jiménez-Hornero et al., (2010) en Córdoba. Por último, Sánchez et al., (2008), y Dueñas et al., (2004) realizaron sendos estudios en la meseta norte y en Andalucía comparando áreas urbanas y rurales.

En el presente estudio se analiza la evolución temporal del ozono en la ciudad de Zaragoza durante el periodo 2002-2008 en el marco de un proyecto de investigación en ciernes que versa sobre la contaminación en la ciudad de Zaragoza y valle del Ebro. Los objetivos de esta primera aproximación son identificar en el periodo de registro sus patrones temporales, y la elaboración de los calendarios promedio diarios y semanales de una ciudad de 600.000 habitantes localizada en el interior de la Península Ibérica y con una elevada insolación. 


\section{Area de estudio}

La ciudad de Zaragoza, situada en el centro de la Depresión del Ebro a orillas de los ríos Ebro, Huerva, y Gállego, tiene un clima mediterráneo semiárido, con fuerte amplitud térmica, característica de las zonas de interior, y un régimen de precipitaciones equinoccial. Las temperaturas promedio de invierno rondan los $4^{\circ} \mathrm{C}$ mientras en verano se alcanzan los $24^{\circ} \mathrm{C}$. Las precipitaciones anuales superan los $320 \mathrm{~mm}$, pero las variaciones interanuales son muy acusadas. Una marcada estación seca en verano no obsta para que sin embargo se puedan producir tormentas con altos valores de precipitación. Por otro lado las precipitaciones mensuales dependen de un elevado peso porcentual de los días de máximo aporte, de manera que existe una acusada concentración en el tiempo de las mismas. Tres características finales del clima de Zaragoza son relevantes en los estudios de contaminación: su alto valor de insolación, al menos durante el periodo seco (primavera-verano), su carácter ventoso (predominando la componente NW-W, el conocido cierzo) y la frecuencia de nieblas invernales al amparo de situaciones anticiclónicas reforzadas con inversiones térmicas en el fondo de la depresión.

En el presente estudio se analizan los datos facilitado por el Ayuntamiento de Zaragoza, durante el periodo comprendido entre los años 2002 y 2008, en tres de las estaciones remotas de la red municipal, a saber: Renovales, El Picarral y Roger de Flor. La elección de estos observatorios del conjunto que mantiene el Ayuntamiento es que son las tres estaciones de referencia de la ciudad en la red de observaciones de la Unión Europea. Su localización se muestra en la Figura 1. Los datos tratados son promedios de doce horas, correspondiendo a una valoración diurna (08-20 horas) y nocturna (20-08 horas) a partir de las medidas horarias originales.

\section{Materiales y Métodos}

\subsection{Sistema de medición}

El sistema de medición que se aplica en la red de seguimiento de inmisiones de la ciudad de Zaragoza consiste en la toma de muestras de aire y su filtrado para evitar la acumulación de partículas en el dispositivo de medición. Dicha muestra se bifurca en dos canales. La muestra de aire del canal uno pasa a través de un catalizador, que elimina el ozono contenido en la muestra. El aire en ausencia de ozono de este canal se utiliza como patrón en la celda de referencia. La muestra de aire del otro canal pasa directamente a la celda de medida. Ambas celdas son irradiadas por un haz de radiación ultravioleta, producida por una lámpara UV de mercurio de baja 


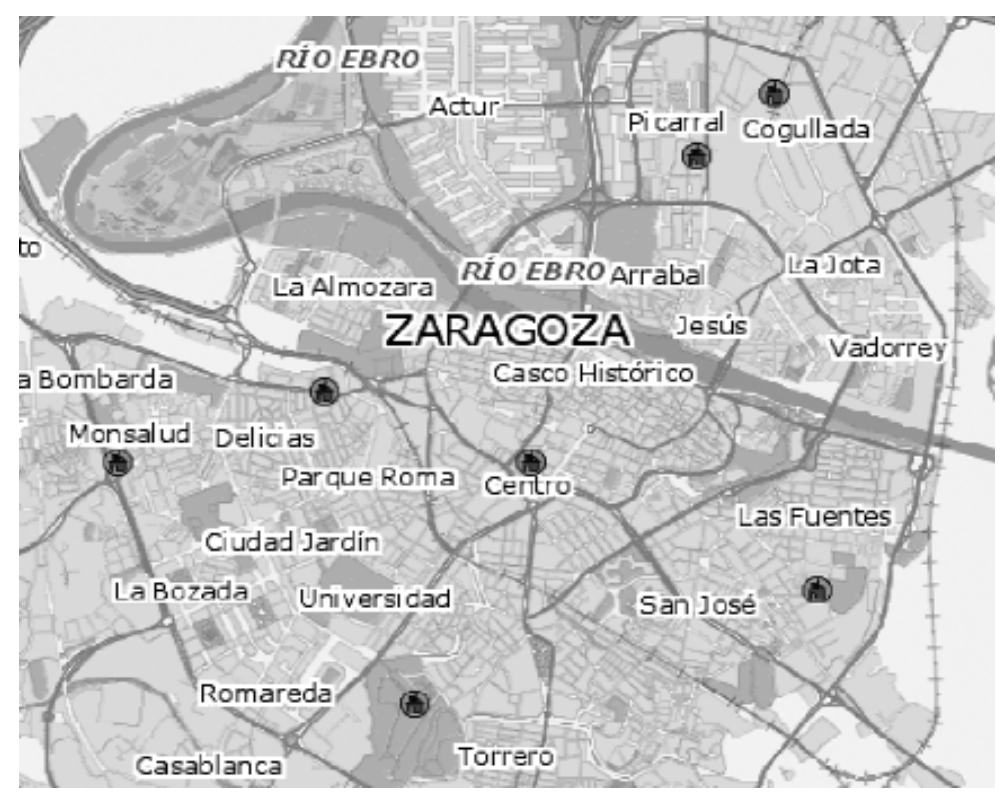

Figura 1. localización muestreos.

presión. La absorbancia producida en la celda de medida, es medida por un tubo fotomultiplicador que suministra una señal eléctrica. El mismo proceso es aplicado a la celda de referencia. La diferente entre la señal del detector de medida y el de referencia es la absorción producida por el ozono. Un circuito resuelve la ecuación de Beer-Lambert y calcula la concentración de ozono en $\mu \mathrm{g} \mathrm{m}^{-3}$.

\subsection{Tratamiento de datos}

Los datos de concentraciones de inmisión entre 2002 y 2008 fueron sometidos a un control de calidad previo eliminando valores anómalos y sospechosos, cuyo origen podría deberse a factores diversos, entre ellos rotura y descalibrado de equipos de medida de inmisión, cortes de luz, etc, entre otros.

Con el fin de detectar las características fundamentales de la serie temporal de las inmisiones de ozono en Zaragoza la base de datos depurada fue sometida a un Análisis de Varianza factorial, considerando como factores el día de medición, el registro diurno-nocturno, el mes y el año. El estudio se completó con la realización de calendarios climáticos (Soler y Martín-Vide, 2002) de los promedios diarios y sema- 
nales, tanto de los valores diurnos como nocturnos. Para su representación gráfica en algunos casos los datos diarios fueron promediados mensual o semanalmente. En el presente estudio no se tratan las diferencias espaciales de los distintos sectores de la ciudad.

\section{Resultados}

El modelo univariado de ANOVA factorial indica que existen diferencias significativas $(\mathrm{p}<0.01)$ entre los valores anuales, entre las series diurnas y nocturnas, y entre meses.

\subsection{Evolución plurianual}

La serie promedio plurianual del ozono en Zaragoza muestra el patrón cíclico característico asemejándose a una onda sinusoidal, cuya máxima amplitud se presenta entre los meses más calurosos de julio y agosto coincidiendo con los momentos de máxima radiación solar, máxima temperatura y mínima nubosidad (véase más adelante), y sus mínimos en los meses de invierno. En la Figura 2 se muestra la evolución de los promedio mensuales diurnos entre 2002 y 2008 y los valores promedio de radiación mensual obtenidos de Solar Radiation Research Laboratory (http://www.nrel.gov/midc/srrl_bms/), donde se puede observar que la relación entre ambas variables es significativa y muy clara $(\mathrm{r} 0.76, \mathrm{p}<0.001)$. En la citada figura también se observa que la amplitud de onda ha crecido en el periodo de estudio, desde máximos en torno a $30 \mu \mathrm{g} \mathrm{m} \mathrm{m}^{-3}$ en los años 2002-2004 hasta máximos que superan los $50 \mu \mathrm{g} \mathrm{m}^{-3}$ en los años 2007-2008, es decir la concentración de ozono en Zaragoza parece haber aumentado durante el periodo de estudio.

\subsection{Los promedios diarios y mensuales}

El calendario de los valores promedio diarios (diurnos y nocturnos) se muestra en la Figura 3. Los valores de concentración oscilan entre aproximadamente mínimos de $10 \mu \mathrm{g} \mathrm{m} \mathrm{m}^{-3}$, y máximos de 40 y $55 \mu \mathrm{g} \mathrm{\textrm {m } ^ { - 3 }}$ en las series nocturna y diurna. En la Tabla 2 se ofrecen los valores promedio mensuales de ozono de Zaragoza. Los valores máximos se dan en los meses de julio y agosto, tanto de día como en la noche y los mínimos en invierno. Las diferencias máximas entre el día y la noche (expresadas en la ratio día/noche o sus diferencias) se producen en periodos de fuerte radiación y temperaturas elevadas (meses de verano), lo que sugiere que la destrucción nocturna en verano es más intensa que en invierno, y que las diferencias se aminoran en los meses de invierno. En la serie destaca un segundo pico máximo en abril que se 


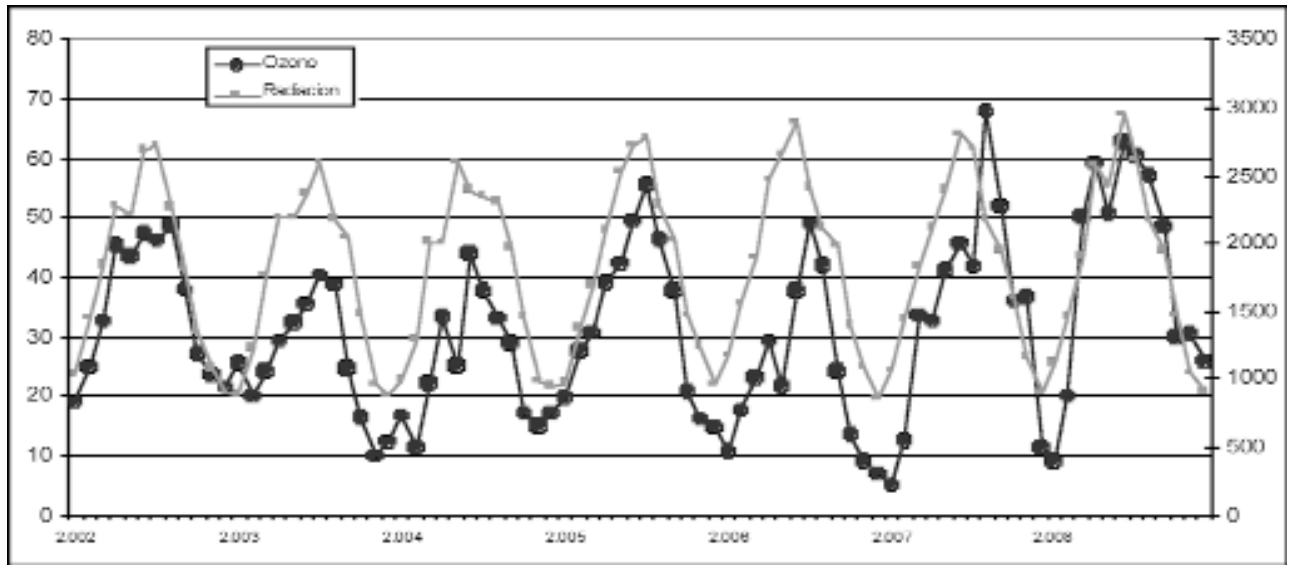

Figura 2. Evolución interanual.

detecta por el aparente descenso de mayo. La Figura 3 incluye los valores promedio diarios de nubosidad obtenidos a partir de la base de datos European Climate Asessement (Klein-Tank et al., 2002). Los mínimos valores de nubosidad de Julio se corresponden con los máximos valores registrados de ozono, de este modo el descenso de nubosidad en los meses de máxima concentración se debe relacionar con el aumento de la reacción fotónica que facilita el incremento de ozono en estos meses.

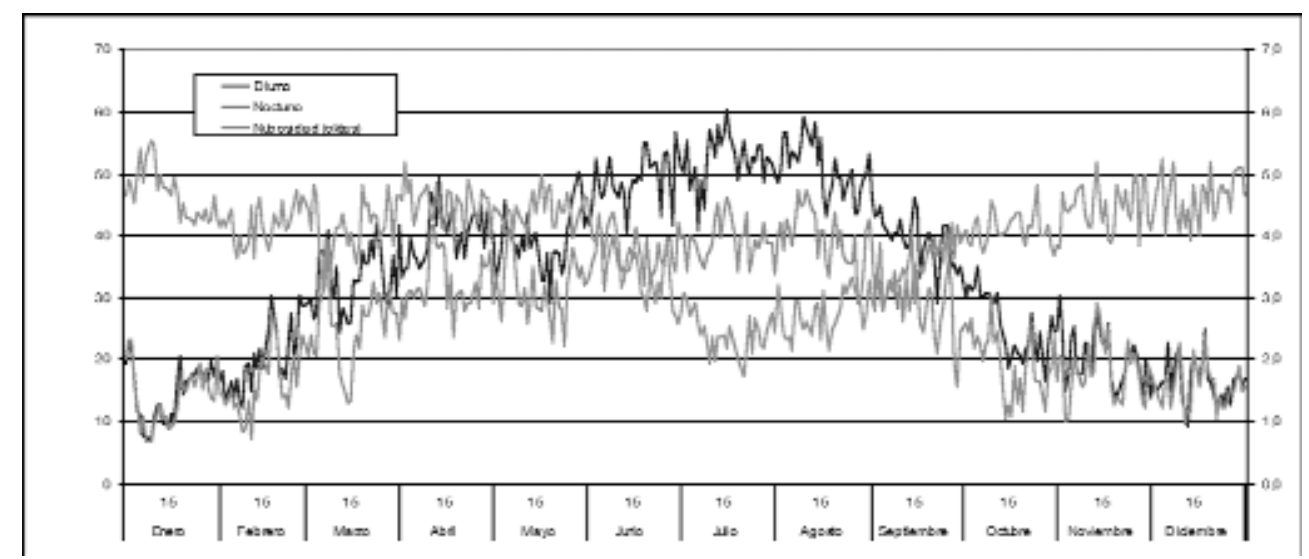

Figura 3. Calendario diario. 
Tabla 2. Promedios mensuales de las inmisiones de Ozono $\left(\mu \mathrm{g} / \mathrm{m}^{-3}\right)$ en Zaragoza, 2002-2008.

\begin{tabular}{|l|c|c|c|c|c|c|c|c|c|c|c|c|}
\hline & $E$ & $F$ & $M Z$ & $A B$ & $M Y$ & $J N$ & $J L$ & $A G$ & $S$ & $O$ & $N$ & $D$ \\
\hline Día & 15,5 & 21,4 & 34,9 & 42,2 & 40,6 & 52,4 & 53,9 & 53,3 & 41,4 & 25,9 & 21,3 & 16,1 \\
Noche & 14,8 & 16,9 & 27,1 & 34,4 & 32,7 & 39,7 & 40,6 & 42,2 & 31,2 & 20,0 & 19,0 & 15,3 \\
Diferencia & 0,716 & 4,414 & 7,823 & 7,842 & 7,904 & 12,66 & 13,27 & 11,12 & 10,21 & 5,94 & 2,275 & 0,828 \\
Ratio & 1,048 & 1,261 & 1,289 & 1,228 & 1,241 & 1,318 & 1,327 & 1,264 & 1,327 & 1,297 & 1,12 & 1,054 \\
\hline
\end{tabular}

\subsection{Los promedios semanales}

Junto a los calendarios de los promedios diarios y promedios mensuales, el calendario semanal proporciona nueva información sobre la estructura en el tiempo de las inmisiones de ozono. Los valores promedio semanales de lunes a domingo de las concentraciones de ozono se muestran en la Tabla 3.

Tabla 3. Promedios diarios semanales de Ozono ( $\mu \mathrm{g} / \mathrm{m}-3)$ en Zaragoza, 2002-2008.

\begin{tabular}{|c|c|c|c|c|c|c|c|}
\hline & Lunes & Martes & Miércoles & Jueves & Viernes & Sábado & Domingo \\
\hline Día & 31,1 & 31,3 & 32,0 & 32,6 & 32,8 & 36,4 & 38,7 \\
Noche & 27,7 & 26,1 & 25,7 & 26,6 & 26,7 & 26,4 & 28,3 \\
Diferencia & 3,4 & 5,2 & 6,3 & 6 & 6,1 & 10 & 10,4 \\
Ratio & 1,12 & 1,20 & 1,25 & 1,23 & 1,23 & 1,38 & 1,37 \\
\hline
\end{tabular}

De lunes a viernes las concentraciones diurnas de ozono aumentan suavemente oscilando entre 31.1 y $32.8 \mu \mathrm{g} \mathrm{m}^{-3}$ y de modo notorio el sábado y domingo sobrepasan $35 \mu \mathrm{g} \mathrm{m} \mathrm{m}^{-3}$. La evolución nocturna sin embargo muestra diferencias menos acusadas que las diurnas y no supera el valor de $30 \mu \mathrm{g} \mathrm{m}^{-3}$. El sábado presenta concentraciones semejantes al resto de la semana y solamente la noche del domingo se destaca entre el resto de días. La diferencia día noche es claramente superior en sábado y domingo al resto de los días de la semana y sugiere que la destrucción de ozono diurno o su transporte es un proceso notable en la ciudad de Zaragoza. En la Figura 4 se muestran los valores diarios promedio de una semana tipo. El ligero valor superior que se observa en la noche "promedio de los lunes" respecto a las noches de martes a sábado podría atribuirse al efecto de arrastre que se produce en la lectura desde el domingo o bien al complejo proceso de mezcla entre capas atmosféricas (Adame et al., 2010; Sánchez et al., 2008). 


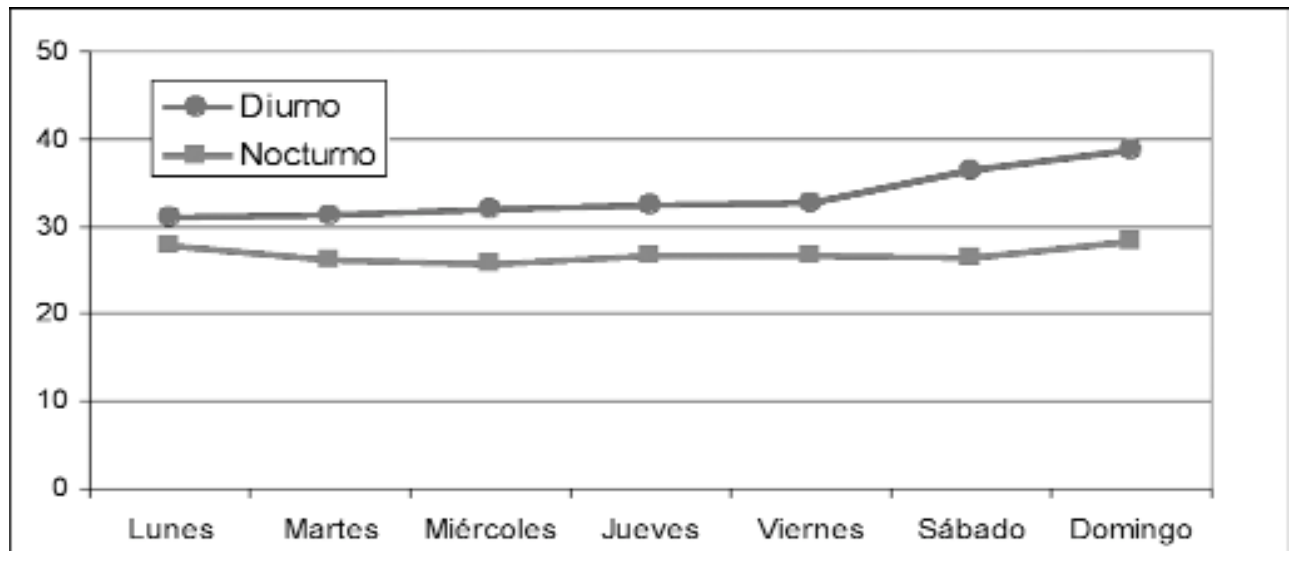

Figura 4. Calendario semanal. Valores promedio diurnos y nocturnos de Ozono $\left(\mu \mathrm{g} / \mathrm{m}^{-3}\right)$ en Zaragoza (2002-2008).

\section{Discusión}

Los valores de concentración de ozono no son estáticos en el tiempo y presentan unos ciclos bien conocidos tanto diarios como estacionales que aparecen claramente en la ciudad de Zaragoza durante el periodo 2002-2008. Dicho patrón tiene una forma sinusoidal de máximos veraniegos y mínimos invernales. El origen de estas variaciones estacionales se debe a que el ozono necesita para su formación radiación solar y temperaturas altas, lo cual se produce sobre todo en los meses de julio y agosto (Figura 2). De la misma manera, el descenso de nubosidad presentado en la Figura 3 sustenta esta afirmación, pues la relación entre nubosidad y radiación recibida en el suelo es inversa. Los máximos veraniegos de concentraciones de ozono en Zaragoza coinciden con las observaciones de Castell et al., (2004a) en la Comunidad Valenciana, quienes atribuyeron las diferencias entre los máximos primaverales de la costa y veraniegos del interior a las diferencias de transporte incrementadas durante los meses de mayor insolación, y han sido registrados en la Península Ibérica en otras localidades (Adame et al., 2010).

La variación de los promedios mensuales de Zaragoza oscila 14 y $50 \mu \mathrm{g} \mathrm{m}^{-3}$, semejantes en magnitud a los descritos por Sánchez-Lorenzo (2004) en Barcelona, y por Baldano et al. (2003) en ciudades europeas, pero son netamente inferiores a los observados en áreas de fuertes emisiones como Puertollano (Saiz-Lopez et al., 2009), en el entorno de Huelva tanto en ambientes rurales como urbanos (Adame e al., 2010), y también en ambientes rurales de la meseta norte (Sánchez et al., 2008). No 
obstante la diferencia entre los máximos y mínimos supera la descrita en el SW espa-

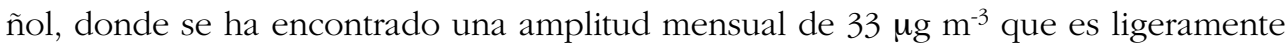
inferior a la encontrada en Zaragoza $\left(>40 \mu \mathrm{g} \mathrm{m}^{-3}\right)$. En consecuencia, la serie promedio de ozono de Zaragoza destaca tanto por sus bajos valores bajos como por las elevadas diferencias entre máximos y mínimos. Estos valores contrastan sobre todo si se compara con las concentraciones de los ambientes rurales citados, en donde se supone que los valores de ozono por la falta de precursores deben tener un origen netamente fotoquímico y ser más bajos que en áreas urbanas (Sánchez et al., 2008). En el presente trabajo no hemos analizado las condiciones meteorológicas específicas paralelas a la serie de inmisiones, pero el carácter ventoso de la ciudad como dispersante de las eventuales concentraciones originadas pudiera ser una explicación plausible que debe ser analizada en detalle en ulteriores investigaciones. La ausencia de ciudades o centros emisores cercanos provocaría que Zaragoza se comportara eventualmente como foco emisor de ozono sobre su entorno. La ampliación de la red de seguimiento a puntos de control en su entorno podría ayudar a explicar esta cuestión.

La evolución de la concentración de ozono en Zaragoza muestra un incremento de la amplitud de onda en los años finales de la serie y como consecuencia una tendencia positiva. Dicha tendencia ha sido observada en España en la meseta norte en el periodo 2000-2006 por Sánchez et al. (2008). La tendencia, calculada en una serie tan corta, no supone admitir su proyección y la ausencia de información posterior a 2008 plantea el interrogante de su futuro. Cabe pensar que tras el periodo de brightning observado al menos hasta 2004 (Sánchez-Lorenzo et al., 2009), el descenso de emisión solar haya producido también un descenso de concentración de ozono, aunque esta hipótesis debe ser contrastada con las series de datos a partir de 2008 pues la presencia de precursores pudiera haber producido un cierto control sobre los valores del contaminante estudiado pese a disminuir la radiación.

Los valores promedio semanales de ozono en Zaragoza son semejantes y con una pauta muy similar a los descritos en Barcelona por Sánchez- Lorenzo (2004) y Jiménez et al. (2005). La hipótesis que se ha sugerido a estos efectos de fin de semana descritos en otras partes es que los aumentos de la concentración de ozono en sábado y domingo se deberían a la disminución de otros contaminantes precursores por el descenso del tráfico y las actividades industriales, a la par que ello comportaría además un aumento de la limpieza atmosférica y radiación. La explicación de esta hipótesis vendría dada por la siguiente cadena de reacciones:

- Entre los contaminantes primarios generados por actividades industriales y tráfico están los $\mathrm{NO}_{x}$, que de acuerdo a su reactividad química son capaces promover el contaminante que es objeto de este estudio: $\mathrm{NO}(\mathrm{g})+\mathrm{O}_{2}(\mathrm{~g}) \longrightarrow$ $\mathrm{NO}_{2}(\mathrm{~g})$. 
- A su vez el dióxido de nitrógeno, por acción de la luz solar, se descompone en monóxido: $\mathrm{NO}_{2}(\mathrm{~g})+\operatorname{luz}($ radiación $) \longrightarrow \mathrm{NO}(\mathrm{g})+\mathrm{O}(\mathrm{g})$

- El monóxido de nitrógeno, puede volver a oxidarse para formar otra vez dióxido de nitrógeno y hacer que el proceso vuelva a comenzar. Pero, el oxígeno atómico es una especie muy reactiva que puede provocar muchas reacciones importantes, una de ellas es la formación de ozono.: $\mathrm{O}(\mathrm{g})+\mathrm{O}_{2}(\mathrm{~g}) \longrightarrow \mathrm{O}_{3}(\mathrm{~g})$

Por último, el análisis de las series temporales de Zaragoza, tanto en la perspectiva plurianual (2002-2008), como promedio diario y semanal permite afirmar que, a tenor de los umbrales legislativos (véase Tabla 1), la ciudad presenta niveles de contaminación muy bajos de ozono, y pese al incremento que se ha observado en el periodo de estudio, en todo momento los registros han quedado siempre por debajo de los límites que la legislación establece para este contaminante tanto para la salud humana como los ecosistemas.

\section{Conclusiones}

El estudio de las series de inmisiones de ozono en Zaragoza entre 2002 y 2008 permite afirmar que las concentraciones de este contaminante no suponen un peligro ambiental en la ciudad, pues los máximos valores observados nunca han rebasado los límites establecidos por la legislación. En general las concentraciones de ozono de Zaragoza presentan valores mucho más bajos que los observados en otras localidades de España tanto urbanas como rurales, pero están en las magnitudes de muchas ciudades del planeta.

El análisis de la serie promedio de ozono de permite constatar el patrón estacional que refleja en gran medida el efecto de la radiación solar. En el periodo analizado la concentración de ozono ha aumentado.

Se detecta claramente el efecto de fin de semana en el patrón semanal, con máximos de concentración durante el sábado y domingo, que se podrían relacionar con la disminución de sus precursores y la limpieza atmosférica.

Adame JA, Bolivar JP and de la Morena BA (2010). Surface ozone measurements in the southweast of the Iberian Peninsula (Huelva, Spain). Environmental Science Pollution Research 17, 355-368.

Baldasano JM, Valera and Jiménez P (2003). Air quality data from large cities. Science of Total Environment 307, 141-165.
Castell N, Mantilla E, Fernández F y López E (2004a). Distribución espacial y temporal de los episodios de contaminación por ozono y su relación con las situaciones sinópticas en la vertiente mediterránea. En JC García Cordrón, C Diego, P Fernández, C Garmendia y D Rasilla (Eds) El clima entre el mar y la montaña. Publ. AEC, Serie A, 4, 599-605 
Castell N, Mantilla E, Fernández F y López E (2004b). Comparación de la variabilidad temporal de los niveles de ozono en un entorno costero y en un entorno interior. En JC García Cordrón, C Diego, P Fernández, C Garmendia y D Rasilla (Eds) El clima entre el mar y la montaña. Publ. AEC, Serie A, 4, 590-598.

Dueñas C, Fernández MC, Cañete S, Carretero J, Liger E (2002). Assessement of ozone variations and meteorological effects in an urbana rea in the Mediterranean coast. Science of Total Environment 299, 97-113.

Dueñas C, Fernández MC, Cañete S, Carretero J, Liger E (2004) Analyses of ozone in urban and rural sites in Malaga. Chemosphere 56, 631-639.

Fernández Garcia, F (2005) Contaminación atmosférica y calidad del aire en Madrid: análisis de las concentraciones de $\mathrm{SO}_{2}$, CO, Ozono y PM10 (1980-2003). Revista de Estudios Geográficos 507- 532.

Garcia MA, Sánchez ML, Pérez IA, de la Torre B (2005). Ground level ozone concentration at a rural location in northern Spain Science of Total nvironment 348, 13491361.

Gerasopoulos E, Kouvarakis G, Vrekoussis M, Donoussis C, Mihalopoulos N, Maria M (2006). Photochemical ozone production in the $\mathrm{E}$ astern Mediterranean. Atmos. Environment 40, 3057-3069.

Ibarra G and Madariaga I (2003). Traffic congestion and ozone precursor emission in Bilbao (Spain). Env. Science Pollution Research 10, 361-367.

Jiménez-Hornero F, Gutiérrez E, Ariza AB and Giraldez JV (2010). Description of the seasonal pattern in ozone concentration time series by using the strange attractor multifractal formalism. Environmental Monitoring Assessement 160, 229-236.
Jiménez P, Parra R, Gassó S , Baldasano JM (2005). Modelling the ozone weekend effect in verycomplex terrains, a case studying the Northeastern Iberian Peninsula. Atmospheric Environment 39, 429-444.

Klein-Tank, A.M., Winjgaard, J.B., Können, G.P. et al., (2002) Daily dataset of 20thcentury surface air temperature and precipitation series for the European Climate Assessment. Int. Journal of Climatology, 22, 1441-1453.

Millán M, Sanz MJ, Salvador , Mantilla E (2002). Atmosphere dynamics and ozone cycles related to nitrogen deposition in the western Mediterranean. Environmental Pollution 118, 167-186.

Palacios M, Kirchnner F, Martili A, Clappier A, Martín F, Rodríguez S (2002;). Summer ozone episodes in the Greater Madrid area. Atmos. Environment 36, 5323-5333.

Saiz-López A, Adame JA, Notario A, Pobrete J, Bolívar JP and Albaladejo J (2009). Yearround observations of $\mathrm{NO}, \mathrm{NO} 2, \mathrm{O} 3, \mathrm{SO} 2$, and Toluene measured with DOAS system in the industrial area of Puertollano, Spain. Water Air Soil Pollution 200, 277-288.

Sánchez ML, de Torre B, García MA, Pérez IA (2007). Ground level ozone and ozone vertical profile measurements close to the foothills of the Guadarrama mountain range (Spaiin). Atmos. Environment 41, 1302-1314.

Sánchez ML, García MA, Pérez IA and de Torre B (2008). Evaluation of surface ozone measurements during 2000-2005 at a rural area in the upper Spanish plateau. J. Atmospheric Chemistry 60, 137-152.

Sánchez-Lorenzo A (2004). Variabilidad de las máximas concentraciones de contaminantes a lo largo de los días de la semana en Barcelona. En JC García Cordrón, C Diego, P Fernández, C Garmendia y D Rasilla 
(Eds) El clima entre el mar y la montaña. Publ. AEC, Serie A, 4, 703-713.

Sanchez-Lorenzo A, Calbo J, Brunetti $M$ and Deser C (2009) Dimming/brightening over the Iberian Peninsula, Trends in sunshine duration and cloud cover and their relations with atmospheric circulation.
Journal of Geophysical Research, Vol. 114, D00D09, doi,10.1029/2008JD011394.

Soler X y Martín Vide J (2002) Los calendarios climáticos, una propuesta metodológica. En JA Guijarro, M Grimalt, M Laita y S Alonso (2002) El Agua y el Clima. Publicaciones, SEC, Ser A, 3, 577-586. 